\title{
Cognitive dysfunction in pediatric multiple sclerosis
}

\author{
This article was published in the following Dove Press journal: \\ Neuropsychiatric Disease and Treatment \\ 23 July 2014 \\ Number of times this article has been viewed
}

\author{
Agnese Suppiej' \\ Elisa Cainelli ${ }^{1,2}$ \\ 'Child Neurology and Clinical \\ Neurophysiology, Pediatric University \\ Hospital, Padua, Italy; ${ }^{2}$ Lifespan \\ Cognitive Neuroscience Laboratory \\ (LCNL), Department of General \\ Psychology, University of Padua, Italy
}

Abstract: Cognitive and neuropsychological impairments are well documented in adult multiple sclerosis (MS). Research has only recently focused on cognitive disabilities in pediatric cases, highlighting some differences between pediatric and adult cases. Impairments in several functions have been reported in children, particularly in relation to attention, processing speed, visual-motor skills, and language. Language seems to be particularly vulnerable in pediatric MS, unlike in adults in whom it is usually preserved. Deficits in executive functions, which are considered MS-specific in adults, have been inconsistently reported in children. In children, as compared to adults, the relationship between cognitive dysfunctions and the two other main symptoms of MS, fatigue and psychiatric disorders, was poorly explored. Furthermore, data on the correlations of cognitive impairments with clinical and neuroimaging features are scarce in children, and the results are often incongruent; interestingly, involvement of corpus callosum and reduced thalamic volume differentiated patients identified as having a cognitive impairment from those without a cognitive impairment. Further studies about pediatric MS are needed in order to better understand the impact of the disease on brain development and the resulting effect on cognitive functions, particularly with respect to different therapeutic strategies.

Keywords: central nervous system, child, deficit, IQ, inflammatory demyelination, neuropsychological

\section{Background}

Multiple sclerosis (MS) is an autoimmune-mediated disorder that causes demyelination of the central nervous system and secondary axonal degeneration, leading to brain atrophy. This chronic inflammatory neurodegenerative disease is typically diagnosed in young to middle-aged adults, but it can occur in childhood, although children and adolescents represent an uncommon patient population, accounting only for $2 \%-5 \%$ or less of patients suffering from MS. ${ }^{1}$

An open question is whether pediatric MS shares the same disease features as adult MS. Clinically, the presentation and course of pediatric and adult MS may be different, particularly when onset is at younger ages. However, it is not clear if these differences are due to pathophysiology of the disease or to the disease processes being expressed on a different substrate (ie, the immature central nervous system and immune system). ${ }^{2}$ Inflammation is more pronounced in pediatric MS, which is characterized by higher rates of relapses, ${ }^{3}$ higher T2 lesion volume observed through magnetic resonance imaging (MRI), ${ }^{4}$ and an often polyfocal onset of symptoms $;{ }^{3}$ moreover, a small proportion of cases fulfill, at onset, criteria for acute disseminated encephalomyelitis, but are later diagnosed as MS. ${ }^{5}$ Compared to adults, neurologic, motor, and cognitive outcomes of children suffering from MS differ in evolution and features,
Correspondence: Agnese Suppiej

Child Neurology and Clinical

Neurophysiology, Pediatric

University Hospital, via Giustiniani, 3,

35100 Padua, Italy

Tel +394982I 8094

Email suppiej@pediatria.unipd.it
Neuropsychiatric Disease and Treatment 2014:10 1385-1392

1385

Dovepress

http://dx.doi.org/1 0.2147/NDT.S48495 (c) (1) (5) 2014 Suppiej and Cainelli. This work is published by Dove Medical Press Limited, and licensed under Creative Commons Attribution - Non Commercial (unported, v3.0) BY LC License. The full terms of the License are available at http://creativecommons.org/licenses//by-nd/3.0/. Non-commercial uses of the work are permitted without any further permission from Dove Medical Press Limited, provided the work is properly attributed. Permissions beyond the scope of the License are administered by Dove Medical Press Limited. Information on
how to request permission may be found at: http://www.dovepress.com/permissions.php 
suggesting different cerebral involvement or enhanced recovery and repair mechanisms, but also differences in immune-pathophysiology. ${ }^{2}$

In this context, the neurocognitive outcome of pediatriconset MS is particularly complex to understand and to predict. Pediatric age is characterized by ongoing myelination in the central nervous system; thus, inflammatory demyelination occurring in MS may result in atypical or incomplete formation of white matter pathways. The integrity of the white matter is a necessary prerequisite for development of neural networks and cognition; moreover, it is not clear if increased plasticity, typical of the immature central nervous system, has a protective role from the deleterious impact of the disease in children. ${ }^{6}$ Demyelination effects and plasticity mechanisms have to be evaluated in the context of timing of development of cognitive functions, as well as in terms of their possible interference with the acquisition of the basic building blocks that are critical for academic achievement. ${ }^{7}$ Taking into account all these aspects, it is not surprising that the cognitive and neuropsychological profile in pediatric MS is similar to, but does not overlap with, adult MS.

Few review articles have been published so far on cognition in pediatric MS; the most comprehensive is that of Blaschek et al. ${ }^{8}$ This work revised major studies on cognitive dysfunction in pediatric MS, published until 2010, and reported frequent deficits in attention, processing speed, and memory. Cognitive deficits seem to emerge early and are generally more severe in children with an early-onset disease. Other important studies, reporting wide numbers of pediatric MS patients, have been subsequently published but are not included in available reviews. ${ }^{6,9-16}$ Moreover, the focus of recent research has included correlations of cognitive dysfunction with MRI abnormalities and clinical features.

The aim of the present study was to perform a comprehensive narrative review. We underwent a systematic search of all articles indexed in PubMed Central, PsychINFO, ScienceDirect, Web of Science, and Scopus, up to January 15, 2014, without time or other limits. The following keywords were used: pediatric OR childhood OR juvenile OR adolescents AND multiple sclerosis AND cognitive OR neuropsychological OR impairments OR outcome. We also inspected the reference list of the retrieved articles to ensure a wider search.

\section{Cognitive dysfunction in pediatric $M S$}

Cognitive dysfunction in pediatric MS was an unexplored field until the last decade, but, in the last few years, research on global cognitive and neuropsychological profiles of children suffering from MS expanded rapidly. Reported impairments occur in a range from $30 \%$ to $80 \%$ of the cases; evaluation criteria are not homogeneous among studies, accounting for some differences in the reported prevalence.

The intelligence quotient (IQ), which is a measure of global cognition, even if abnormally low in some patients, is generally better preserved than neuropsychological function where a wide range of deficits - including attention, information processing speed, memory, executive functions, and some aspects of language and visual-motor domains have been reported.

\section{Data on global cognitive functioning in pediatric $M S$}

The full IQ profile was evaluated by a complete administration of the Wechsler Intelligence Scale in only a few studies; however, a short form of the Scale, the Wechsler Abbreviated Scale of Intelligence, has been frequently used to integrate an extensive neuropsychological assessment.

The overall mean group IQ was in the range expected from published age-stratified normative data, ${ }^{6,7,9,10,17-19}$ but a lower mean group IQ emerged by comparing MS patients with matched healthy controls..$^{6,20,21}$ Furthermore, 8\%-30\% of the individual patients had an IQ $<70 .^{6,21,22}$

\section{Data on neuropsychological functions in pediatric MS Attention and information processing}

Most studies evaluating the attention domain considered several components of this function and used the Trail Making Test, the Symbol Digit Modalities Test, or the Continuous Performance Test. Complex aspects of attention, such as shifting attention between complex stimuli, were the more compromised components. When tested, attention and information processing were found to be abnormal in nearly all studies; deficits appeared to emerge very early in the course of the disease. Impairments were particularly evident from comparison of MS patients' performance with that of healthy controls. $^{7,9,11,18,21}$

\section{Visual-motor and visual-spatial skills}

The majority of data on visual-motor and visual-spatial skills in pediatric MS come from studies using tests such as the Visual Motor Integration test and the Rey Figure. Impairments, both on visual-motor integration skills and on visual memory, are often reported (eg, spatial recall ${ }^{23}$ and immediate visual recall ${ }^{24}$ ). 


\section{Memory}

Deficits in verbal and visual memory, both immediate and delayed as well as in episodic and working memory, have been reported in pediatric and juvenile patients. . $^{12,20,21,24}$

\section{Language}

In pediatric MS, an involvement of language is frequently seen. Children are still developing complex linguistic skills; therefore, language seems to be particularly vulnerable in this age group. By contrast, language dysfunctions seem relatively rare in adult-onset MS, and linguistic problems are usually associated with more generalized cognitive impairment. Indeed, differences in linguistic involvement appear to be the main neuropsychological difference between children and adults with MS.

The linguistic profile of pediatric MS was studied with tests evaluating different aspects of expressive and receptive language. The most used tests were the Vocabulary and Similarities tests of the Wechsler Intelligence Scale, verbal fluency, and naming tests. Juvenile MS patients seem to have poor performance in complex and speed-dependent language functions, ${ }^{18,24}$ while naming skills appear to be less affected. Tests of expressive language, although rarely compromised, are useful to discriminate between children suffering from MS and healthy matched controls. ${ }^{6,718}$

\section{Executive functions}

The most-used tests in studies evaluating the executive functions in MS patients were planning and abstract reasoning tasks, such as the Wisconsin Card Sorting test and the Tower of London test. In adults, particular deficits in conceptual reasoning are considered to be specific to $\mathrm{MS}^{-25-27}$ by contrast, in pediatric MS, conceptual reasoning is not commonly impaired. However, executive functions encompass multiple interdependent abilities (ie, working memory, attention shifting), which are more commonly affected in children as an expression of executive-function involvement in a different stage of brain maturation. ${ }^{13,21,24}$ In many affected children, neuropsychological impairments may compromise intellectual functions and academic performance..$^{20,21}$ Deficit in attention, information processing speed, and executive functions may have an impact on activities such as listening to lengthy instructions, organization of unstructured assignments, and generation of novel ideas. These skills are increasingly needed in higher academic grades and correlate with the clinical observation of declining school performance.

Furthermore, pediatric MS patients appear to show a selective vulnerability in mathematic skills despite average
IQ and the provision of unlimited time to complete mathematic questions. ${ }^{14}$ However, some measures of academic achievement (reading and spelling) may be completed within age expectations, demonstrating that the use of measures of academic achievement alone, similarly to measures of global intellectual function, may miss or underestimate the real cognitive impairments. ${ }^{6,20,28}$

Up until now, most studies in literature calculated an index of cognitive impairment from a complete neuropsychological assessment. This global measure of cognitive functioning was calculated from impairment of single functions; criteria for classification of impairment differed widely between studies. For some authors, dysfunction was based on at least two impaired cognitive tasks ${ }^{24,28}$ while, for others, dysfunction was based on at least three impaired cognitive tasks, ${ }^{6,11,21,22}$ or five or more tests. ${ }^{9}$ Finally, others graded the cognitive performance based on the percentage of abnormal functions. ${ }^{18}$ This methodology is useful to quantify a wide range of impairments in a unitary measure, but it may introduce important differences in results, leading to studies that are barely comparable.

Thus, research does not yet provide extensive data on cognitive evaluation of pediatric MS using a standardized validated neuropsychological battery specifically tailored for this age range. However, brief and cost-effective validated instruments have been recently proposed internationally.

\section{Time course of cognitive dysfunction in pediatric MS}

Research in adults suffering from MS converged to show a progression of cognitive loss over time, both in patients cognitively intact at baseline and in those initially showing some degree of impairment. ${ }^{29,30}$ Most of the studies on pediatriconset MS report cognitive dysfunctions often appear early in the course of the disease, close to MS diagnosis. Furthermore, impairments are more severe in those with younger age at disease onset. ${ }^{6,21}$ Information about the long-term outcome of cognitive deficits is scarce. , $^{911,18,20,28,31}$ A lower cognitive functioning was reported in children with more recent disease onset (within 12 months) compared to those with later onset (mean 5 years); more widespread deficits, involving mainly tasks requiring self-generated organizational strategies or reliance on efficient processing speed or working memory, were seen in those with longer disease duration. ${ }^{20}$ Similar results were found by MacAllister et $\mathrm{al}^{28}$ who described a deterioration over time between the first cognitive evaluation (nearly 22 months from MS onset) and evaluation at follow up (12-18 months later). In the study by Amato et $\mathrm{al}^{18} \mathrm{a}$ further cognitive decline over time, or impairment following 
previously normal functioning, was found in the majority of the pediatric patients tested after 5 years from first evaluation (which was done at MS onset). The deterioration involved most of the tested cognitive measures; in particular, verbal memory, complex attention, verbal fluency, and receptive language.

In pediatric neurodegenerative disorders, the deleterious effects of disease progression on cognitive abilities coexist with expected maturational improvement in function, creating more difficulty in detecting evolution, particularly at the individual level. Indeed, a recent study that used a robust statistical method to determine individual changes on cognitive tests showed improvement in functioning on only $18 \%$ of the measures in the MS group as compared with $86 \%$ of the measures in the control group. These results highlight a lack of expected maturational improvements in the MS patients relative to age-matched healthy peers. Deterioration in functioning, defined as significant decline on three or more tests, was observed in $25 \%$ of the patients, whereas more global deterioration, defined as decline on five or more tests, was observed in roughly $11 \%$ of the patients. ${ }^{9}$ Differences between studies may be due to more stringent cut-off, different statistical approach, or simply differences in the timing of follow-up.

\section{Psychiatric disorders in pediatric MS}

Among psychiatric disorders, depression is a common symptom in MS, with a prevalence ranging from $27 \%$ to $54 \%$ in adults. ${ }^{32}$ Depression might originate from prolonged hospitalization or be the result of the psychological impact of the MS diagnosis itself; on the other hand, the fact that patients seem to be more affected by depressive states than other neurologic patients with and without involvement of the central nervous system suggests that depression may represent a primary symptom of $\mathrm{MS} .{ }^{33-35}$ A relationship between depression and immunomodulatory treatment was also suggested, but results are incongruent. ${ }^{33,36,37}$

The prevalence of affective disorders in children suffering from MS varies from $6 \%$ to $46 \%,{ }^{20,21,24,31,38}$ probably due to the different assessment strategies (Children's Depression Inventory, Kiddie-SADS-Present and Schedule for Affective Disorders and Schizophrenia for School-Age-ChildrenPresent and Lifetime Version [K-SADS-PL]). Although many studies evaluated the prevalence of affective symptoms in children with MS, the correlation with cognitive and neuropsychological impairment remains a less-explored field. The only study fully addressing this question is that of
Weisbrot et al. ${ }^{15}$ They found a variety of psychiatric diagnoses among 45 pediatric MS patients undergoing psychiatric assessment. Out of the 25 children with a psychiatric disorder, more than two-thirds had more than one psychiatric diagnosis. The most frequent were anxiety disorders, mood disorders, and attention deficit hyperactivity disorder. No differences were found in patients with or without a psychiatric disorder with respect to cognitive impairment, but children with concurrent mood or anxiety disorders had a higher frequency of cognitive dysfunction when compared with children with other psychiatric diagnoses. The authors suggested an influence of depression and anxiety symptoms on attention and information-processing skills, but they could also be independent manifestations of the underlying demyelinating central nervous system disease.

\section{Fatigue in pediatric MS}

Fatigue is among the most common symptoms of MS. In adults, the frequency ranges from $65 \%$ to $95 \%$ across studies $^{39,40}$ and many patients with MS describe fatigue as their most disabling symptom, significantly interfering with daily functioning. ${ }^{41}$ Fatigue has been defined as an overwhelming sense of tiredness, lack of energy, and feeling of exhaustion of such severity as to interfere with usual and desired activities. Despite its significance, the pathogenesis of fatigue is poorly understood.

The prevalence and severity of fatigue, and its complex relationship with cognitive dysfunction, have been extensively investigated in the literature on adult-onset MS. However, to date, there is limited information on the impact of fatigue on cognitive impairment in children and adolescents with MS. The majority of studies considered fatigue as part of the evaluation of cognitive dysfunction or depression. 7,18,21,24,42,43 There is a heterogeneity of instruments used to test fatigue and often it is measured from simple dichotomous yes/no questions or with the Fatigue Severity Scale, which is validated for adults. When tested in children and adolescents, this scale provided low sensitivity levels in the identification of fatigue. MacAllister et $\mathrm{al}^{24}$ failed to show an effect of fatigue on cognitive profile. Amato et $\mathrm{al}^{21}$ did not find differences in fatigue between patients with and without cognitive impairment, even if fatigue was reported as a common symptom.

Recently, a Multidimensional Fatigue Scale has been proposed, entailing items from the Pediatric Quality of Life Inventory administered both to children and their parents. ${ }^{44}$ Goretti et al ${ }^{11}$ correlated the Pediatric Quality of Life Inventory-Multidimensional Fatigue Scale subjective reports of cognitive and other fatigue-related aspects with 
occurrence of neuropsychological impairments and psychiatric disorders. They failed to find an association between the overall measure of subjectively assessed cognitive fatigue and global cognitive performance. However, higher levels of self-reported cognitive fatigue by children were associated with impaired performance on a problem-solving test, while higher levels of parent-reported cognitive fatigue were associated with impairment on tests of verbal learning, processing speed, complex attention, and verbal comprehension.

\section{Clinical features predicting cognitive impairment in pediatric $\mathbf{M S}$}

Research on clinical risk factors for cognitive decline are very scarce; the factors most frequently considered are age at onset, MS duration and number of relapses, severity of neurological disability, and effect of therapy.

\section{Age at onset}

Few studies evaluated the effect of age at onset of MS on cognitive outcome, and results are incongruent between studies. ${ }^{6,18,20,24}$ Amato et al found that low IQ scores were significantly associated with younger age at MS onset; they suggested that earlier neuropathological damage in the central nervous system can have more disruptive impact on the development of intellectual abilities. ${ }^{21}$ However, by evaluating the same MS patients 2 years later, the effect of age at MS onset disappeared. ${ }^{18}$ Banwell and Anderson ${ }^{20}$ found a more severe cognitive impairment in a group of children with earlier onset of MS compared to a group with later onset; since they tested both groups at the same age it could not be determined if the effect was due to longer duration of the disease or earlier age at onset. Indeed, other authors showed that association of more severe cognitive dysfunction with younger age at disease onset disappeared after controlling for disease duration. ${ }^{6,24}$

\section{MS duration and number of relapses}

Data in the literature are scarce and fail to demonstrate an effect of disease duration on cognitive outcome. . $^{611,18,20,24,28}$ The relapsing-remitting form of MS, where episodes of neurologic dysfunction are followed by a period of recovery, appears to be very common in childhood MS, with estimates ranging from $93 \%$ to $100 \%$ of the cases. ${ }^{45-48}$ However, similar to disease duration, the number of relapses does not seems to influence cognitive decline, ${ }^{11,18}$ or the influence was too weak to be noticeable. ${ }^{24,28}$

\section{Neurological disability}

Neurologic data in MS patients were usually collected using the Expanded Disability Status Scale (EDSS). The EDSS is a ten-point scale of neurologic impairment, quantifying eight functional systems: pyramidal; cerebellar; brainstem; sensory; bowel/bladder; visual; cerebral; and other. A summary scale is generated that rates impairment according to a scale ranging from zero - indicating normal neurological function - to ten - indicating death due to MS. ${ }^{49}$ Over the first 15 years of MS, EDSS scores tend to be less than three in the large majority of in pediatric patients. However, some studies indicated neurological disability as the most robust predictor of cognitive impairment in children suffering from MS. A hierarchical multiple regression analysis used to evaluate the relative contribution of several clinical variables in predicting cognitive outcome (EDSS, number of relapse, MS duration, age at onset) showed that EDSS was the strongest predictor; ${ }^{24}$ moreover, a neurological impairment emerging early in the course of the disease was predictive of cognitive decline. ${ }^{16,28}$ These findings are consistent with results in adult-MS literature pointing out an association between EDSS and cognitive loss. ${ }^{29}$

In contrast with the above studies, other authors reported that cognitive deficit occurred independently from neurological disability as assessed with EDSS, likely reflecting the relatively limited accrual of physical disability early in the MS disease course of pediatric-onset MS patients. ${ }^{6,11,18,20}$

\section{Therapy}

Studies in adults suggest that disease-modifying drugs may positively influence the cognitive outcome of the patient in the long-term. ${ }^{50}$ The effects of these treatments on cognitive outcome has not been specifically tested in pediatric MS; however, up to $90 \%$ of the children included in studies on cognitive outcome of pediatric MS were receiving diseasemodifying drugs. When studies attempted to correlate cognitive outcome with therapy, among a variety of other risk factors, no effect was found. ${ }^{18}$ Furthermore, while studying the effect of therapy on cognitive functioning, it should be taken into account that treatment with interferon beta has been linked to the occurrence of depression in adults with MS. ${ }^{51}$

\section{MRI correlates of cognitive impairment in pediatric MS}

In adults with MS, cognitive impairment has been positively associated with several MRI abnormalities: axonal and 
neuronal damage, as measured by global brain atrophy; ${ }^{52-54}$ cortical volume; ${ }^{55}$ ventricular enlargement; ${ }^{55-57}$ and thalamic atrophy. ${ }^{58,59}$ Other associations were also suggested but results were not always replicated. ${ }^{6}$ Spatial distribution of lesions showed little correlation with specific cognitive deficits, as highlighted by the incongruent results reported in studies investigating the relationship between executive deficits and frontal lobe lesions. ${ }^{60-62}$ However, it has been speculated that the pathological processes in MS may be more diffuse in nature, given the occurrence of changes also in the so called "normal appearing white matter". ${ }^{63}$

Less is known regarding the cognitive impact of MRI lesions on pediatric MS; studies specifically linking cognitive dysfunction to neuroimaging in childhood MS are scarce, but there is emerging evidence of some MRI correlates of neuropsychological deficits.

Research showed that, in children, patterns of lesion distribution slightly differed from that of adults, probably due to incomplete myelination of anterior regions. In children, lesions were mainly reported in the pons, peduncles, brainstem, and cerebellum. ${ }^{6466}$ Selective loss of volume of the thalami compared to healthy controls was reported in a study of 28 pediatric-onset MS patients, ${ }^{67}$ but the cognitive correlations were not described.

Till et $\mathrm{al}^{6}$ evaluated neuropsychological performance and MRI correlates in 34 pediatric-onset MS patients and in 33 demographically age-matched healthy children. They identified a cognitive impairment in $29 \%$ of the patients, predominantly involving attention and processing speed, expressive language, and visual-motor integration; MRI showed significantly lower thalamic volume, total brain volume, and gray matter volume in MS children. Interestingly, corpus callosum and thalamic volume differentiated patients identified as having a cognitive impairment from those without. Furthermore, thalamic volume was the most robust MRI predictor of global IQ, processing speed, and expressive vocabulary scores, though corpus callosum area, normalized brain volume, and T2-weighted lesion volume were also significantly correlated with most cognitive outcomes. About 15 months later, 28 of the MS patients and 26 of the healthy controls were retested in order to evaluate changes of cognitive functioning in time. Surprisingly, changes in brain volume and lesion volume did not predict changes in cognitive status, with the exception of a significant relationship between increased lesion volume and slower psychomotor speed on attention tasks. $^{9}$

\section{Conclusion}

Research over the last few years has improved our understanding of the impact of childhood MS on cognitive functions. Recent data on large series suggests that deficits occur early in the course of the disease and affect children's quality of life. Language, an early developing function, appears particularly vulnerable in this age group.

In children, as opposed to adults, the degenerative processes occur before the full maturation of cognitive abilities; therefore, failure to show age-expected progress is considered a sign of disease progression.

The relationship of cognition with the other two main symptoms, psychiatric disorders and fatigue, is beginning to also be appreciated in pediatric MS.

The predictive value of clinical and neuroimaging features on cognitive outcome has not been extensively explored, but emerging data suggest a relationship between regional brain volume (particularly thalamic volume) measured on MRI and neuropsychological findings.

A limitation of the present review was the lack of a systematic search. Furthermore, comparison of available studies has been hampered by the differences in adopted protocols and the variability of criteria used to define abnormalities. However, brief and cost-effective validated instruments for evaluation of cognitive functions in pediatric MS have been proposed recently. ${ }^{7}$

Future research should include multicenter studies with agreed protocols for testing cognitive function and common criteria for definition of abnormalities. More longitudinal studies with multiple assessment points and long-term evaluation, ideally up to adulthood, are needed. They should take into account resiliency factors such as the baseline social and cultural status as well as preexisting school performance.

Important directions for future research will be the identification of risk factors for cognitive decline by correlation of patients' clinical and instrumental data with cognitive outcome; individual neuroimaging indicators of cognitive reserve should be included when evaluating the time-course of cognitive decline. Research should also take into account the evaluation of the effect of different disease-modifying agents on cognitive performance.

The clinical implications of such studies for potential interventions and rehabilitation strategies, ultimately addressed to improve children's quality of life, cannot be overlooked.

\section{Disclosure}

The authors report no conflicts of interest in this work. 


\section{References}

1. Chitnis T, Glanz B, Jaffin S, Healy B. Demographics of pediatric-onset multiple sclerosis in an MS center population from the Northeastern United States. Mult Scler. 2009;15:627-631.

2. Chitnis T. Paediatric MS is the same disease as adult MS: No. Mult Scler. 2013;19(10):1255-1256.

3. Gorman MP, Healy BC, Polgar-Turcsanyi M, Chitnis T. Increased relapse rate in pediatric-onset compared with adult-onset multiple sclerosis. Arch Neurol. 2009;66:54-59.

4. Yeh EA, Weinstock-Guttman B, Ramanathan M, et al. Magnetic resonance imaging characteristics of children and adults with paediatriconset multiple sclerosis. Brain. 2009;132:3392-3400.

5. Banwell B, Bar-Or A, Arnold DL, et al. Clinical, environmental, and genetic determinants of multiple sclerosis in children with acute demyelination: A prospective national cohort study. Lancet Neurol. 2011;10: 436-445.

6. Till C, Ghassemi R, Broche-Aubert B, et al. MRI correlates of cognitive impairment in childhood-onset multiple sclerosis. Neuropsychology. 2011;25(3):319-332.

7. Portaccio E, Goretti B, Lori S, et al. The brief neuropsychological battery for children: a screening tool for cognitive impairment in childhood and juvenile multiple sclerosis. Mult Scler. 2009;15(5):620-626.

8. Blaschek A, Storm van's Gravesande KS, Heinen F, Pritsch M, Mall V, Calabrese P. Neuropsychological aspects of childhood multiple sclerosis: an overview. Neuropediatrics. 2012;43:176-183.

9. Till C, Racine N, Araujo D, et al. Changes in cognitive performance over a 1 -year period in children and adolescents with multiple sclerosis. Neuropsychology. 2013;27(2):210-219.

10. Smerbeck AM, Parrish J, Serafin D, et al. Visual-cognitive processing deficits in pediatric multiple sclerosis. Mult Scler. 2011;17(4): 449-456.

11. Goretti B, Portaccio E, Ghezzi A, et al. Fatigue and its relationships with cognitive functioning and depression in paediatric multiple sclerosis. Mult Scler. 2012;18(3):329-334.

12. Fuentes A, Collins DL, Garcia-Lorenzo D, et al. Memory performance and normalized regional brain volumes in patients with pediatric-onset multiple sclerosis. J Int Neuropsychol Soc. 2012;18(3): 471-480.

13. Till C, Ho C, Dudani A, García-Lorenzo D, Collins DL, Banwell BL. Magnetic Resonance Imaging Predictors of Executive Functioning in Patients with Pediatric-Onset Multiple Sclerosis. Arch Clin Neuropsychol. 2012;27:1-15.

14. Till C, Deotto A, Tipu V, et al. White matter integrity and math performance in pediatric multiple sclerosis: a diffusion tensor imaging study. Neuroreport. 2011;22(18):1005-1009.

15. Weisbrot D, Charvet L, Serafin D, et al. Psychiatric diagnoses and cognitive impairment in pediatric multiple sclerosis. Mult Scler. 2014;20(5): 588-593.

16. Julian L, Serafin D, Charvet L, Ackerson J, Ralph B. Cognitive impairment occurs in children and adolescents with multiple sclerosis: results from a United States network. J Child Neurol. 2013;28(1): 102-107.

17. McCann KK, Farmer JE, Patel N. Childhood-onset multiple sclerosis and mood disorders: A case study. Child Neuropsychol. 2004;10: 102-116.

18. Amato MP, Goretti B, Ghezzi A, et al. Cognitive and psychosocial features in childhood and juvenile MS: two-year follow-up. Neurology. 2010;75:1134-1140.

19. Marin S, Banwell BB. Cognitive trajectories in 4 patients with pediatriconset multiple sclerosis: serial evaluation over a decade. J Child Neurol. 2013;28(12):1577-1586

20. Banwell BL, Anderson PE. The cognitive burden of multiple sclerosis in children. Neurology. 2005;64(5):891-894.

21. Amato MP, Goretti B, Ghezzi A, et al. Cognitive and psychosocial features of childhood and juvenile MS. Neurology. 2008;70(20):1891-1897.

22. Lori S, Portaccio E, Zipoli V, et al. Cognitive impairment and eventrelated potentials in paediatric multiple sclerosis: 2-year study. Neurol Sci. 2011;32:1043-1046.
23. Amato MP, Ponziani G, Pracucci G, Bracco L, Siracusa G, Amaducci L. Cognitive impairment in early-onset multiple sclerosis. Pattern, predictors, and impact on everyday life in a 4-year follow-up. Arch Neurol. 1995;52:168-172.

24. MacAllister WS, Belman AL, Milazzo M, et al. Cognitive functioning in children and adolescents with multiple sclerosis. Neurology. 2005;64(8): $1422-1425$.

25. Rao SM. Neuropsychology of multiple sclerosis: a critical review. J Clin Exp Neuropsychol. 1986;8(5):503-542.

26. Petersen RC, Kokmen E. Cognitive and psychiatric abnormalities in multiple sclerosis. Mayo Clin Proc. 1989;64(6):657-663.

27. Penman MF. Assessing the prevalence of cognitive impairment in multiple sclerosis: implications for patient management. Axone. 1991;13(2): 45-49.

28. MacAllister WS, Christodoulou C, Milazzo M, Krupp LB. Longitudinal neuropsychological assessment in pediatric multiple sclerosis. Dev Neuropsychol. 2007;32(2):625-644.

29. Amato MP, Ponziani G, Siracusa G, et al. Cognitive dysfunction in early-onset multiplesclerosis: A reappraisal after 10 years. Arch Neurol. 2001;58:1602-1606.

30. Kujala P, Portin R, Ruutiainen J. The progress of cognitive decline in multiple sclerosis. A controlled 3-year follow-up. Brain. 1997;120: 289-297.

31. Goretti B, Ghezzi A, Portaccio E, et al. Psychosocial issue in children and adolescents with multiple sclerosis. Neurol Sci. 2010;31(4): 467-470.

32. Minden SL, Schiffer RB. Affective disorders in multiple sclerosis. Review and recommendations for clinical research. Arch Neurol. 1990; 47(1):98-104.

33. Whitlock FA, Siskind MM. Depression as a major symptom of multiple sclerosis. J Neurol Neurosurg Psychiatry. 1980;43(10): $861-865$.

34. Schiffer RB, Babigian HM. Behavioral disorders in multiple sclerosis, temporal lobe epilepsy, and amyotrophic lateral sclerosis. An epidemiologic study. Arch Neurol. 1984;41(10):1067-1069.

35. Rabins PV, Brooks BR, O'Donnell P, et al. Structural brain correlates of emotional disorder in multiple sclerosis. Brain. 1986;109 (Pt 4):585-597.

36. Patten SB, Metz LM. Interferon beta-1 a and depression in relapsingremitting multiple sclerosis: an analysis of depression data from the PRISMS clinical trial. Mult Scler. 2001;7:243-248.

37. Arnett PA, Randolph JJ. Longitudinal course of depression symptoms in multiple sclerosis. J Neurol Neurosurg Psychiatry. 2006;77(5): 606-610.

38. Till C, Udler E, Ghassemi R, Narayanan S, Arnold DL, Banwell BL. Factors associated with emotional and behavioral outcomes in adolescents with multiple sclerosis. Mult Scle. 2012;18(8): $1170-1178$

39. Fisk JD, Pontefract A, Ritvo PG, Archibald CJ, Murray TJ. The impact of fatigue on patients with multiple sclerosis. Can J Neurol Sci. 1994; 21(1):9-14.

40. Lobentanz IS, Asenbaum S, Vass K, et al. Factors influencing quality of life in multiple sclerosis patients: disability, depressive mood, fatigue and sleep quality. Acta Neurol Scand. 2004;110(1):6-13.

41. Krupp LB, Alvarez LA, LaRocca NG, Scheinberg LC. Fatigue in multiple sclerosis. Arch Neurol. 1988;45:435-437.

42. MacAllister WS, Christodoulou C, Troxell R, et al. Fatigue and quality of life in pediatric multiple sclerosis. Mult Scler. 2009;15(12): $1502-1508$.

43. Ketelslegers IA, Catsman-Berrevoets CE, Boon M, et al. Fatigue and depression in children with multiple sclerosis and monophasic variants. Eur J Paediatr Neurol. 2010;14(4):320-325.

44. Varni JW, Burwinkle TM, Szer IS. The PedsQL Multidimensional Fatigue Scale in pediatric rheumatology: reliability and validity. J Rheumatol. 2004;31:2494-2500.

45. Pinhas-Hamiel O, Sarova-Pinhas I, Achiron A. Multiple sclerosis in childhood and adolescence: Clinical features and management. Paediatr Drugs. 2001;3(5):329-336. 
46. Boiko A, Vorobeychik G, Paty D, Devonshire V, Sadovnick D. Early onset multiple sclerosis: a longitudinal study. Neurology. 2002;59(7): 1006-1010.

47. Ghezzi A, Pozzilli C, Liguori M, et al. Prospective study of multiple sclerosis with early onset. Mult Scler. 2002;8(2):115-118.

48. Simone IL, Carrara D, Tortorella C, et al. Course and prognosis in early-onset MS: Comparison with adult-onset forms. Neurology. 2002;59(12):1922-1928.

49. Kurtzke JF. Rating neurologic impairment in multiple sclerosis: an expanded disability status scale (EDSS). Neurology. 1983;33: 1444-1452.

50. Fischer JS, Priore RL, Jacobs LD, et al. Neuropsychological effects of interferon beta-1a in relapsing multiple sclerosis. Multiple Sclerosis Collaborative Research Group. Ann Neurol. 2000;48:885-892.

51. Lublin FD, Whitaker JN, Eidelman BH, et al. Management of patients receiving interferon beta- $1 \mathrm{~b}$ for multiple sclerosis: report of a consensus conference. Neurology. 1996;46:12-18.

52. Rao SM, Leo GJ, Haughton VM, St Aubin-Faubert P, Bernardin L. Correlation of magnetic resonance imaging with neuropsychological testing in multiple sclerosis. Neurology. 1989;39:161-166.

53. Benedict RH, Carone DA, Bakshi R. Correlating brain atrophy with cognitive dysfunction, mood disturbances, and personality disorder in multiple sclerosis. J Neuroimaging. 2004;14:36S-45S.

54. Calabrese M, Agosta F, Rinaldi F, et al. Cortical lesions and atrophy associated with cognitive impairment in relapsing-remitting multiple sclerosis. Arch Neurol. 2009;66:1144-1150.

55. Benedict RH, Bruce JM, Dwyer MG, et al. Neocortical atrophy, third ventricular width, and cognitive dysfunction in multiple sclerosis. Arch Neurol. 2006;63:1301-1306.

56. Benedict RH, Bakshi R, Simon JH, Priore R, Miller C, Munschauer F. Frontal cortex atrophy predicts cognitive impairment in multiple sclerosis. J Neuropsychiatry Clin Neurosci. 2002;14:44-51.
57. Sánchez MP1, Nieto A, Barroso J, Martín V, Hernández MA. Brain atrophy as a marker of cognitive impairment in mildly disabling relapsingremitting multiple sclerosis. Eur J Neurol. 2008;15(10):1091-1099.

58. Blinkenberg M, Rune K, Jensen CV, et al. Cortical cerebral metabolism correlates with MRI lesion load and cognitive dysfunction in MS. Neurology. 2000;54:558-564.

59. Houtchens MK, Benedict RH, Killiany R, et al. Thalamic atrophy and cognition in multiple sclerosis. Neurology. 2007;69:1213-1223.

60. Swirsky-Sacchetti T, Mitchell DR, Seward J, et al. Neuropsychological and structural brain lesions in multiple sclerosis: A regional analysis. Neurology. 1992;42:1291-1295.

61. Arnett PA, Rao SM, Bernardin L, Grafman J, Yetkin FZ, Lobeck L. Relationship between frontal lobe lesions and Wisconsin Card Sorting Test performance in patients with multiple sclerosis. Neurology. 1994; 44:420-425.

62. Foong J, Rozewicz L, Davie CA, Thompson AJ, Miller DH, Ron MA. Correlates of executive function in multiple sclerosis: The use of magnetic resonance spectroscopy as an index of focal pathology. J Neuropsychiatry Clin Neurosci. 1999;11:45-50.

63. Dousset V, Grossman RI, Ramer KN, et al. Experimental allergic encephalomyelitis and multiple sclerosis: Lesion characterization with magnetization transfer imaging. Radiology. 1992;182:483-491.

64. Ghezzi A, Deplano V, Faroni J, et al. Multiple sclerosis in childhood: Clinical features of 149 cases. Mult Scler. 1997;3:43-46.

65. Simone IL, Carrara D, Tortorella C, Ceccarelli A, Livrea P. Early onset multiple sclerosis. Neurol Sci. 2000;21(4 Supp1 2):S861-S863.

66. Balassy C, Bernert G, Wöber-Bingöl C, et al. Long-term MRI observations of childhood-onset relapsing-remitting multiple sclerosis. Neuropediatrics. 2001;32:28-37.

67. Mesaros S, Rocca MA, Absinta M, et al. Evidence of thalamic gray matter loss in pediatric multiple sclerosis. Neurology. 2008;70(13 Pt 2): 1107-1112.
Neuropsychiatric Disease and Treatment

\section{Publish your work in this journal}

Neuropsychiatric Disease and Treatment is an international, peerreviewed journal of clinical therapeutics and pharmacology focusing on concise rapid reporting of clinical or pre-clinical studies on a range of neuropsychiatric and neurological disorders. This journal is indexed on PubMed Central, the 'PsycINFO' database and CAS,

\section{Dovepress}

and is the official journal of The International Neuropsychiatric Association (INA). The manuscript management system is completely online and includes a very quick and fair peer-review system, which is all easy to use. Visit http://www.dovepress.com/testimonials.php to read real quotes from published authors. 